\title{
36
}

\section{Impact of Organisational Maturity on Software Quality}

\author{
Margaret Tan ${ }^{a}$ and Chee Yuen Yap ${ }^{b}$ \\ a Department of Decision Sciences, National University of Singapore, Kent Ridge Crescent, \\ Singapore 0511 \\ b Acer Computer International Pte Ltd, Singapore
}

\begin{abstract}
Introducing quality process improvements in software development is a challenge. The organisation requires a certain level of maturity to undertake a quality management programme. Often such programme involves a process re-engineering paradigm. It also embodies a new culture that encourages relentless and continuous improvement of the way all the activities are executed. An exploratory study investigating the impact of organizational maturity on software quality practices is conducted on 280 companies in Singapore. The objective of the study is to understand the issues underlying the implementation of software quality management concepts.
\end{abstract}

Keyword Codes: D.2.9; K.6.3; K6.4

Keywords: Software Engineering, Management; Software Management; System Management

\section{INTRODUCTION}

Software quality is very important with increasing application of information technology to critical areas like medicine, safety, and security. Poor quality software is no longer tolerable. Customers are now shifting their attention from prices at the time of purchase to life cycle costs that include expenditures on services and maintenance - the total costs.

Because software engineering has evolved into a highly complex process with its high impact on the organization, the only way to incorporate quality is to manage it on a corporate-wide basis that reaches every level in the organization. However, the organisation that introduces quality management must have a sophisticated level of maturity. A maturity level where there is strategic planning and a mission definition with multi-year implementation techniques.

The traditional software development environment where quality of the software rests in the hands of individual developers is inadequate. Software developers have realized that quality cannot be effectively and efficiently attained by only the developers. All parties involved in the software development processes must be motivated and committed to quality. Quality has to be "built in" to be coordinated in the management processes. In other words, it must be "managed in" as part of strategic planning and not merely as a "control in" function [1].

The purpose of this study is to see how rigorous IT companies in Singapore are in adopting a disciplined process to software development. The objective is also to understand the issues underlying the implementation of software quality process management concepts. This study uses the Software Engineering Institute (SEI) software capability maturity model (CMM) [2] as a yardstick to measure the organisational maturity in adopting good software development practices.

\section{SOFTWARE QUALITY MANAGEMENT}

Quality is defined as "both a philosophy and a set of guiding principles that represent the foundation for a continuously improving organization. ...... (and which) integrates fundamental management techniques, existing improvement efforts, and technical tools under a disciplined approach focused on continuous improvement" [3]. This definition encompasses an organization-wide relationship of people, 
technologies, processes, and management to work towards customer satisfaction. It embodies a culture of motivating staff through involvement, empowerment, and communication; education, training and learning; improving work processes and systems; instilling management commitment, leadership, and support; and establishing quality assurance policies, objectives and mission. It encourages relentless, continuous improvement of the way all the activities of an organization are executed, the end result of which will lead to products and services of consistent and dependable quality at low cost [4].

Quality process improvement model institutionalizes the systems of making permanent fundamental changes in all processes and activities of the organization to reduce or eliminate waste, to enhance good management practices, and to enrich the working environment to increase management yields. To be successful, it must be part of corporate planning so that there is a sharing of the vision and objectives by all members of the organization. As the organisation needs to be re-structured, and the old processes challenged, the implementation is a multi-year incremental re-engineering design and process. Like any other management initiative, a successful transformation or re-engineering of software development processes need clearly defined objectives, roles, policies, and accountabilities.

The objective of the process-oriented approach is to "build in" quality at every phase of the development cycle and involving every member of the team. Quality should be designed at the beginning of each process and not at the end by inspection (testing) after the product (system) is developed. Final testing does not ensure quality [5]. Failures or errors are much more expensive to fix after the system has been built. Besides, the "quick fix" provides few lasting gains.

\section{SOFTWARE PROCESS MATURITY MODEL}

Before an organisation embarks on software quality planning, it is necessary for it to assess and understand the current state of its software development process. There are many ways in which an organization can assess its state of practice. The SEI software process maturity assessment is one such method. It uses a 120-question assessment model that identifies the organization's capability to plan, define, manage, execute, and improve the software development processes and it then categorizes the organization across five process levels of maturity. By establishing the organisation's position in this framework, management can identify areas where improvement actions will be most effective.

The first level, which is the lowest level is also called the "initial" or "ad hoc" level. This level characterizes an organization as having a laissez-faire approach where development procedures are ill defined. There is no formal or consistent procedure, nor is there a mechanism to ensure systematic procedures are followed. Change control is laxed and there is little understanding on key issues of configuration management nor quality assurance.

The second level, the "repeatable" process or "intuitive" level, characterizes an organization as having a management framework with some practiced sets of methods and tools. In other words, a project management approach maybe used. However, it lacks some process focus and technical standards. The organisation at this level learns from past experience to repeat its future performance.

The third level, the "defined" or "qualitative" level, characterizes the organization as having defined functional processes institutionalized in the use of modem software engineering tools, techniques, and standards. The organization has a systematic and methodological process analysis. However, it has not yet begin to take process data for benchmark improvements.

The fourth level, a fairly advanced level, is called the "managed" or "quantitative" level. At this level processes are well defined and they are quantified, measured, and controlled. An organisation in this level uses a process and statistical database to control and drive software quality. When a process is under a statistical benchmark, repeating the work in roughly the same way will produce a sustained and consistent result.

Finally, the fifth level, the highest level, is called the "optimizing" level. It characterizes an organization as having reached an advanced, sophisticated environment that focuses on rigorous defectcause analysis and prevention for continuous improvement. There is a mechanism to identify the weakest process elements, correct the process to achieve optimum performance.

\section{RESEARCH METHOD}

A field study using survey questionnaire, adapted from the SEI CMM framework, is used to collect data on the organisational maturity of software development practice in Singapore. A pilot study was conducted to test for comprehensiveness and validity. 
The questionnaire is sent out to the chief information officer and it measures the company profile in terms of revenue, size, ownership, age, software development purpose, and number of developers. It also measures the technical and management characteristics of the software development practices.

280 companies in Singapore which have software development either for in-house use or sale to extemal customers participated. The sample is stratified to include software vendors, private enterprises, and govemment organisations.

\section{RESULTS}

The response rate of 45.7 percent consists of 128 responses, out of 280 organisations. However, only 88 responses have software development, thus, giving a valid response rate of 31.4 percent that is used for data analysis. The biggest group of respondents comes from software vendors, representing 42 percent, followed by private enterprises, 34 percent, and finally the government representing 24 percent.

Table 1 shows the distribution of the process maturity levels of the organisation. It is not surprising that most organizations - 72 percent - are in Level 1, the "ad hoc" level. As level 1 characterizes the organization as not having any well-defined procedure and process for development, such organization lacks a mechanism for management reviews in terms of performance tracking and change control. Often the cost and schedule estimates made are unpredictable.

It is interesting to observe that the organisation profile of the responses show that most organizations are fairly young ( 31 percent are less than 8 years old), have small revenue ( 35 percent having less than $S \$ 10$ million), have a small number of developers ( 64 percent of organisations have less than 26 developers). Therefore, it is no surprise that most organizations may not have the necessary sophistication in quality assurance programme. Another plausible explanation may be that most organizations do not have the necessary expertise in terms of resources, or priorities in terms of strategy to understand the structured software development approach. On the other hand, they may not consider it necessary to have a disciplined approach as most of their projects may have fairly narrow scope like projects for modifications and enhancements on existing systems.

Table 1: Distribution of Software Capability Maturity Level

\begin{tabular}{|ccccc|}
\hline $\begin{array}{c}\text { Maturity } \\
\text { Level }\end{array}$ & $\begin{array}{c}\text { Software } \\
\text { Vendor }\end{array}$ & $\begin{array}{c}\text { Private } \\
\text { Enterprise }\end{array}$ & $\begin{array}{c}\text { Government } \\
\text { Organisation }\end{array}$ & Total \\
\hline 1 & 28 & 25 & 19 & 72 \\
2 & 2 & 5 & 3 & 10 \\
3 & 6 & 2 & 2 & 10 \\
4 & 0 & 1 & 0 & 1 \\
5 & 6 & 1 & 0 & 7 \\
\hline
\end{tabular}

The results show that only 10 percent of the organisations are at Level 2 - "repeatable" process that describes an organization as having some structured procedure to software development. However, these organisations lack process standards and definitions to assign process resources. It appears that the majority of this level is represented by private organizations -5 percent, followed by the government - 3 percent. Unfortunately, the vendors are the least represented with only 2 percent. A plausible explanation is that private organizations having higher revenue ( 26 percent having more than $\$ \$ 100$ million), maybe be said to have better management resources and skills to expend greater effort on project management. As the government is the initiator of IT usage since the mid 1980 , it is no surprise that most of their projects would have adopted a fairly well-structured procedure (National Computer Board being the developer of most government computerization programmes).

On the other hand, Level 3 having 10 percent overall representation, is mostly found in vendors - 6 percent. This is followed by equal representation of 2 percent each by the private and government organisations. It is encouraging to see that vendors are having greater awareness and concern for establishing a quality process measurement system that requires identification of quantitative quality goals, plans, tracking, and measurements. It appears that this defined level may not be the priority of private and government organisations if their applications are not of a critical nature. However, in the vendor organization, because software development is the core activity, reaching Level 3 indicates their involvement and commitment in pursuing quality product and services. It is interesting to observe that 5 
percent of organizations reaching this level also have a fairly large number of developers (more than 100 persons). A simple explanation is that with a large number of developers, "building in" quality is necessary to co-ordinate the tasks to enforce the consistency and dependency that is required of the products. Of course, with higher human resources, "organizational decomposition is effective in just the same way as software decomposition" [7]. The organization is able to explore greater avenues of process management, like design and code reviews, change controls, and the use of sophisticated tools and techniques. Organisations with small numbers of developers, on the other hand, constrained by their resources, may be preoccupied with "fire-fighting" techniques of error detection. It must be noted that reaching this level of maturity requires committed resource investment in the quality system.

The higher levels of 4 and 5 , however, are poorly represented. Only 1 percent of the organisation, and surprisingly this comes from the private enterprises, reach level 4 maturity. Level 4 characterizes the organisations as having some reasonable statistical and benchmarking control over product quality. Level 5 characterizes the organization as one that emphasizes continuous process improvements through error-cause analysis. Interestingly, 7 percent of the organisations have attained this level. It is encouraging to see 6 percent of software vendor out of the overall 7 percent embracing Level 5 maturity. Developing software for external customers may require different levels of rigour than developing software for internal customers. Internal customers may be less stringent on the quality than extemal customers who are more likely to be more demanding to ensure the systems developed are to their specifications and satisfaction.

It is not surprising to note that 5 percent of these 7 percent are companies with foreign-equity. It appears that foreign-equity companies are more conscious of the increasing importance of quality management for competitive advantage. It could also represent that there is a well managed software development process in its home country. This trend indicates that software process quality is still at its preliminary stage amongst local equity.

\section{CONCLUSION}

The understanding of quality management goes far beyond a simple concern for the reliability of inspection methods used. Instead, it encourages relentless, never-ending improvement of the way all the activities of an organization are executed. It seeks to provide products and services that satisfy the customers with their consistent and dependable quality, at low cost $[8,9]$. Increased productivity will only come from increased quality at every input to the computer development chain. Good process management techniques may provide the impetus to resolving the software crisis of missed schedules, overrun budgeted, software defects, and increasing backlog vis-a-vis applications demand. Further, it would increase the company's reputation that in turn would increase market share.

The government of Singapore has generated tremendous interests and enthusiasm in encouraging globalization as strategies for Singapore to compete in the 1990s. As such, having quality in its software products and services is a strategic way to compete and sustain its competitiveness. However, quality is a relatively new concept in the software development environment in Singapore, the results are not surprising. To obtain continuous improvement, a formal system of quality planning, auditing, and goal setting is necessary. As most organizations are very weak in understanding and having an organizational structure in terms of its maturity level for a quality process-oriented environment, it is equally no surprise that the elements of quality system concept are poorly understood, what more embraced. It is quite clear that a lot of work is needed to introduce the quality concept in software development.

\section{REFERENCES}

1. Garvin, D.A. Quality on the Line. Harvard Business Review, Sept-Oct 1983, pp 65-75.

2. Humphrey, W.S. Managing the Software Process. Reading, Ma: Addison-Wesley, 1989.

3. Schulmeyer, G.G. Zero Defect Software. New York: McGraw-Hill, 1990.

4. Hames, R.D. Total Quality Management: The strategic advantage. Total Quality Management, $1991,2,1$.

5. Aeh, R.K. Focus on the process. Journal of Systems Management, November 1990, p 20.

6. Humphrey, W.S. and W.L.Street A Method for Assessing the Software Engineering Capability of Contractors, Technical Report, Software Engineering Institute, Carnegie Mellon University, Sept 1987, pp 219-244.

7. Daly, E.B. Organizing for successful software development. Datamation, 1979, pp 107-116.

8. Deming, W.E.Quality, Productivity, and competitive Position. Cambridge: MIT Press, 1982.

9. Feigenbaum, A.V. Total Quality Control. New York: McGraw-Hill, 1986. 